\title{
Religionspolitik in Deutschland nach dem Ende des Sozialismus
}

1803 endete ein fast 1000jähriges System von Kirche und Staat in Deutschland, als Bischöfe und Äbte ihre politische Macht verloren haben, der größte Teil der Kirchengüter verstaatlicht und die meisten Klöster aufgelöst wurden. In der ersten Hälfte des 19. Jahrhunderts kämpfte die katholische Kirche um eine angemessene finanzielle Entschädigung, indem Bischöfe und zum Teil Pfarrer sowie der Unterhalt der Kirchen aus dem Staatshaushalt finanziert werden sollten. Später entledigte sich der Staat der Verpflichtung teilweise, indem er der Kirche die Möglichkeit einräumte, ihre Mitglieder mit seiner Hilfe zu besteuern. ${ }^{2}$ In der zweiten Hälfte des 19. Jh. kam es zum „Kulturkampf“, den der mehrheitlich protestantische preußische Staat gegen die Katholische Kirche nach dem

1 Prof. f. Christliche Gesellschaftslehre an der Kath.- Theol. Fakultät der Ruhr-Universität Bochum, Dr. rer.pol, (Volkswirtschaftslehre), lic. theol. Habilitation in Christliche Sozialwissenschaften, Berater der Caritaskommission der Deutschen Bischofskonferenz. Forschungsschwerpunkte: Politische Ethik, Wirtschaftsethik, Wohlfahrtsverbände und Sozialpolitik.

2 Vgl. zur Entwicklung der deutschen Kirchenfinanzierung: S. Häring, Historische Begründung und Entwicklung der Staatsleistungen an die katholische Kirche in Deutschland bis 1919, in: Grund und Grenzen staatlicher Religionsförderung unter besonderer Berücksichtigung des Verhältnisses von Staat und Katholischer Kirche in Deutschland, hrsg. von M. Pulte, A. Hense (Kirchen- und Staatskirchenrecht 17), Paderborn 2014, S. 27-44. 
Ersten Vatikanischen Konzil führte. Katholische Priester sollten über ein Abitur verfügen, an staatlichen Universitäten ausgebildet werden, sowie ein „Kulturexamen“ ablegen, damit sie mit der modernen - durch die Aufklärung geprägten - Bildung, vertraut wurden. Der Jesuitenorden wurde verboten, das Tragen von Ordenskleidung in der Öffentlichkeit untersagt. Die meisten Bischofssitze in Preußen waren unbesetzt, weil die Bischöfe im Gefängnis saßen oder im Exil lebten. Die Vakanz galt auch für die Mehrheit der Pfarrstellen, die nur mit staatlicher Genehmigung besetzt werden durften. Nach 1887 wurde der Kulturkampf beendet und Bischofsstühle sowie Pfarrstellen wieder besetzt.

Die jeweiligen Evangelischen Landeskirchen in Deutschland waren bis 1919 in der Regel Teil des Staatsapparates der Bundesstaaten des Deutschen Reichs, so dass sie durch den Staat (in Preußen etwa durch das preußische Kultusministerium) verwaltet wurden. Erst nach der Trennung von Kirche und Staat in der Weimarer Reichsverfassung 1919 wurden die ersten evangelischen Bischöfe gewählt und es gab eine kirchliche Selbstverwaltung der evangelischen Landeskirchen. Das Grundgesetz von 1949 übernimmt die Kirchenartikel der Weimarer Reichsverfassung. Es garantiert die Religionsfreiheit. Für religiöse Fragen sind die Bundesländer zuständig, so dass nicht der Bund, sondern jeweils die einzelnen Länder die Staats-Kirchen-Beziehungen, z. B. durch Konkordate mit dem Vatikan bzw. Staatskirchenverträge mit der Ev. Kirche, neuerdings auch durch Religionsverträge mit muslimischen Verbänden, regeln.

Nach dem zweiten Weltkrieg gehörten 95,8\% der Bevölkerung (44,3\% Katholisch, 51,5\% Evangelisch) ${ }^{3}$ einer der beiden christlichen Kirchen an. Da die DDR überwiegend protestantisch war, hatten beide Konfessionen in Westdeutschland etwa die gleiche Mitgliederzahl. Die Zahl der einheimischen Katholiken sank in Westdeutschland aber bereits seit 1954, weil seither die Sterbefälle und Austritte höher waren als die

3 Vgl. Th. Großbölting, Der verlorene Himmel, Glaube in Deutschland seit 1945, Bonn 2013, S. 27. Über kirchliche Entwicklung in Deutschland seit dem 19.Jahrhundert informiert: A. Liedhegener, Säkularisierung als Entkirchlichung. Trends und Konjunkturen in Deutschland von der Mitte des 19. Jahrhunderts bis zur Gegenwart, [in:] Umstrittene Säkularisierung: Soziologische und historische Analysen zur Differenzierung von Religion und Politik, Hg. K. Gabriel u.a., Berlin 2012, S. 481-531. 
Taufen und Eintritte. In den 50er- und 60er-Jahren gab es einen erheblichen katholischen Einfluss auf die Politik. Allerdings konnte die Kirche in zwei Fragen ihre Position nicht durchsetzen: Zum einen wurde 1959 das auf Druck der Katholischen Kirche unter Berufung auf das Naturrecht beschlossene - Familienrecht ${ }^{4}$, welches dem Mann einen Vorrang in der Familie einräumte, vom Verfassungsgericht für verfassungswidrig erklärt. Seither spielt das klassische katholische Naturrecht in der politischen Diskussion in Deutschland keine Rolle mehr. Diese Tradition des katholischen Naturrechts wurde bereits 1963 von Ratzinger ${ }^{5}$ wegen seiner „ideologischen Elemente“ kritisiert, weil es eine „überzeitliche Sozialdogmatik zu formulieren versucht", die es so nicht geben könne.

Ein zweiter Konfliktpunkt war die Schulfrage. ${ }^{6}$ Bereits 1949 bei der Verabschiedung des Grundgesetzes hatten die Bischöfe ihre Position nicht durchgesetzt, wonach staatliche Schulen grundsätzlich Konfessionsschulen (ausschließlich katholisch bzw. protestantisch) sein sollten. In den 60er-Jahren entstand in Deutschland eine breite Bildungsdiskussion, wobei als der am meisten benachteiligte Bevölkerungskreis die katholischen Mädchen der Arbeiterfamilien im ländlichen Raum identifiziert wurden. Die dadurch ausgelöste Debatte führte zur Auflösung kleiner staatlicher katholischer Konfessionsschulen und der Bildung von Gemeinschaftsschulen.

In den 70er-Jahren bestand ein großer Konflikt um die Frage der Freigabe der Abtreibung ${ }^{7}$ in den ersten drei Schwangerschaftsmonaten (Fristenlösung), was die damalige Mehrheit von Sozialdemokraten und Freidemokraten im Bundestag beschlossen hatte. Der anfängliche Versuch des Ratsvorsitzenden der Evangelischen Kirche in Deutschland und des Vorsitzenden der Deutschen Bischofskonferenz, eine öffentliche Debatte und eine politische Entscheidungsfindung über die Liberalisierung

4 Ch. Schönberger, Positivität des Rechts und Naturrecht im katholischen Staatsdenken, [in:] Handbuch der Katholischen Soziallehre, Hg. A. Rauscher, Berlin 2008, S. 801-811, bes. S. 803 (FN 13).

5 J. Ratzinger, Naturrecht, Evangelium und Ideologie in der katholischen Soziallehre, [in:] K. v. Bismarck, W. Dirks, Christlicher Glaube und Ideologie, Mainz 1964, S. 24-31.

6 Vgl. Th. Großbölting, Der verlorene Himmel, Glaube in Deutschland seit 1945, a.a.O., S. $61 \mathrm{f}$. sowie $125 f$.

7 Vgl. Th. Großbölting, Der verlorene Himmel, Glaube in Deutschland seit 1945, a.a.O., S. 69 u. S. 131-136. 
des Abtreibungsrechts in einer autoritativen Weise zu unterbinden, wurde gesellschaftlich rasch als im Widerspruch zur demokratischen Öffentlichkeit stehend zurückgewiesen und beide Kirchen begannen grundrechtlich zu argumentieren. In der Abtreibungsdebatte der 70er Jahre $^{8}$ mobilisierte die katholische Kirche ihre Gläubigen auch in zahlreichen Demonstrationen und machte damit von einem demokratischen Grundrecht Gebrauch. Dieser gesellschaftliche Konflikt wurde entschärft, indem das Verfassungsgericht die völlige Freigabe der Abtreibung in den ersten drei Monaten (Fristenlösung) für verfassungswidrig erklärte. Es wurde eine Indikationslösung eingeführt. Nach der deutschen Einheit kam es zu einer Neuregelung, weil in der DDR eine reine Fristenlösung bestand und eine Rechtsangleichung notwendig wurde. Seit 1993 gelten Abtreibungen zwar weiterhin als rechtswidrig, aber nach einer verbindlichen Beratung wird auf eine Bestrafung verzichtet. Die Beratung soll der werdenden Mutter eine eigenständige Gewissensentscheidung zur Annahme des Kindes ermöglichen, damit nicht der Kindserzeuger, die Familie oder der Arbeitgeber und das soziale Umfeld Druck zur Abtreibung ausüben.

\section{Auswirkungen der Wiedervereinigung für die religiöse Situation}

Die Religionszugehörigkeit in Deutschland hat sich in den letzten Jahrzehnten wie folgt entwickelt.

\begin{tabular}{|l|c|c|c|c|c|}
\hline \multirow{2}{*}{} & \multicolumn{2}{|c|}{ WESTDEUTSCHLAND } & \multicolumn{3}{c|}{ GESAMTDEUTSCHLAND } \\
\cline { 2 - 6 } & 1970 & 1987 & 1990 & 2007 & 2014 \\
\hline Evangelisch & $49 \%$ & $41,6 \%$ & $36,9 \%$ & $30,1 \%$ & $28,9 \%$ \\
\hline Katholisch & $44,6 \%$ & $42,9 \%$ & $35,4 \%$ & $30,2 \%$ & $29,9 \%$ \\
\hline
\end{tabular}

8 Vgl. dazu auch: A. Liedhegener, Macht, Moral und Mehrheiten. Der politische Katholizismus in der Bundesrepublik Deutschland und den USA seit 1960, Baden-Baden 2006, S. 351-357. 


\begin{tabular}{|l|c|c|c|c|c|}
\hline \multirow{2}{*}{} & \multicolumn{2}{|c|}{ WESTDEUTSCHLAND } & \multicolumn{2}{c|}{ GESAMTDEUTSCHLAND } \\
\cline { 2 - 6 } & 1970 & 1987 & 1990 & 2007 & 2014 \\
\hline $\begin{array}{l}\text { Ohne Religions } \\
\text { zugehörigkeit }\end{array}$ & $3,9 \%$ & $11,4 \%$ & $22,4 \%$ & $33,8 \%$ & $34,0 \%$ \\
\hline Muslime & $1,3 \%$ & $2,7 \%$ & $3,7 \%$ & ca. $4 \%$ & $4-5 \%$ \\
\hline
\end{tabular}

(Quelle: Großbölting, S. 184 Zahlen 1970-2007; 2014: Forschungsgruppe Weltanschauungen in Deutschland http://fowid.de/ Zugriff am 10.11.2015).

Die Zahlen von Muslimen werden schlecht erfasst, weil es wegen der Kirchensteuer nur für Protestanten und Katholiken amtliche Erhebungen gibt. Zwar stammen ca. 4,2 Mill. Menschen aus muslimischen Ländern, davon bezeichnen sich ca. $50 \%$ als religiös. Daher geben manche auch nur 2,6 Mill. Muslime an.

Organisierte Konfessionslose gibt es derzeit nur ca. 30.000 (von über 30 Mio. Einwohnern ohne Religionszugehörigkeit). Hindus und Buddhisten haben mit rd. 100000 Mitgliedern auch in der Gegenwart nur eine geringe Bedeutung.

In der DDR gehörten 1949 80\% der Evangelischen und 11\% der Katholischen Kirche an. Bis 1990 sank die Zahl der Katholiken auf 5\%, die der Protestanten auf 20\%.

In der DDR war es nicht zu der größeren Rückkehr in die Kirchen aus dem Personenkreis gekommen, der während des Nationalsozialismus ausgetretenen war. Vielmehr führte der Druck der kommunistischen Regierung dazu, dass ein immer geringerer Anteil einer der beiden großen Kirchen angehörte.

Nach der Wiedervereinigung wurden die Staats-Kirchen-Beziehungen der alten Bundesrepublik weitgehend auf die neuen Bundesländer überführt ${ }^{9}$, obwohl dies vor allem in der Evangelischen Kirche der früheren DDR umstritten war. Dies galt für die Kirchensteuer und Militärseelsorge. Besonders intensiv waren die Konflikte um die Einführung des schulischen,

9 Vgl. A. Liedhegener, Macht, Moral und Mehrheiten. Der politische Katholizismus in der Bundesrepublik Deutschland und den USA seit 1960, a.a.O., S. $182 \mathrm{f}$. 
konfessionellen Religionsunterrichts, weil über $80 \%$ der Schüler ungetauft waren. So gibt es in Berlin und Brandenburg heute keinen staatlichen konfessionellen Religionsunterricht. Historisch bedingt gilt dies auch für die Stadtstaaten Bremen und Hamburg, denen 1949 im Grundgesetz ein Sonderrecht eingeräumt wurde (,Bremer Klausel“).

Seit der deutschen Einheit hat sich folgender Wandel vollzogen:

1. Die Zahl der Mitglieder der Evangelischen und Katholischen Kirche schrumpft, weil es erstens mehr Beerdigungen als Taufen und zweitens eine große Zahl von Kirchenaustritten gibt, der vor staatlichen Institutionen zu erklären ist und dann an die Pfarrämter weitergemeldet wird. So steigt die Anzahl der Konfessionslosen.

2. Die kleinen jüdischen Gemeinden ${ }^{10}$ erhielten eine erhebliche Zuwanderung aus Osteuropa, vor allem Russland. Die massiven Integrationsaufgaben führten dazu, dass der Staat mit den jüdischen Gemeinden Verträge abgeschlossen hat, wonach diese erhebliche Mittel aus Steuergeldern erhalten. 1945 gab es 15000 Juden, heute gibt es 110000 bei jüdischen Gemeinden eingetragene und rd. 90000 nicht praktizierende Personen aus jüdischen Familien.

3. Es kamen mehrere Millionen deutschstämmige Aussiedler aus den ehemaligen sozialistischen Staaten. Darunter waren aus Russland viele christliche freikirchliche Gemeinden. Diese versuchten möglichst geschlossen zusammenzuleben.

4. Die Frage der muslimischen Bevölkerung ist besonders seit den Terroranschlägen von 2001 in die öffentliche Diskussion geraten. Wie sich die verstärkte Zuwanderung von muslimischen Flüchtlingen, vor allem aus Syrien, auswirkt, kann noch nicht abgesehen werden.

Die ersten muslimischen Zuwanderer der 1960er-Jahre haben sich mittlerweile endgültig in Deutschland niedergelassen, indem eine zweite und dritte Generation heranwächst. Knapp die Hälfte der rd. 4-5 Millionen Muslime besitzt bereits die deutsche Staatsangehörigkeit. Die Muslime wollen sich sichtbar (z. B. durch Moscheebau an repräsentativen Stellen statt in Hinterhöfen) etablieren. Diese Entwicklung ruft zwei grundsätz-

10 Th. Großbölting, Der verlorene Himmel: Glaube in Deutschland seit 1945, Göttingen-Bristol, CT 2013, s. 224. 
liche Fragen nach der kirchlichen Einflussnahme in einer pluralistischen Demokratie und nach den etablierten Staats-Kirchen-Beziehungen hervor.

\section{Kirchliche Einflussnahme in pluraler Demokratie und Gesellschaft}

Seit der deutschen Abtreibungsdebatte, stärker aber noch nach der deutschen Einheit, als eine Bevölkerungsmehrheit von über 70\% Ungetaufter in die größere Bundesrepublik hinzukam, mussten sich die christlichen Kirchen über ihren Beitrag zur Willensbildung in der demokratischen Öffentlichkeit vergewissern.

Die biblische Überlieferung, die Tradition der Kirche und ihre aktuelle Lehre haben in der politischen Debatte - genauso wie Stimmen von Bischöfen - keinen Stellenwert, der den Diskurs von vornherein eingrenzt oder beendet, sondern müssen sich argumentativ in der Meinungsbildung der pluralen Öffentlichkeit rechtfertigen. Dabei gelten in Deutschland christliche Kirchen durchaus als moralische Instanzen, deren Beitrag in der öffentlichen Debatte erwünscht ist und die gehört werden. Dazu müssen sich beide Kirchen de facto aber vorab auf gemeinsame Positionen verständigen, was nicht immer gelingt. Für die säkulare Öffentlichkeit entsteht bei unterschiedlichen kirchlichen Positionen der Eindruck, dass die biblischen Schriften verschiedene Auslegungen möglich machen, z. B. wenn in evangelischen Kirchen gleichgeschlechtliche Paare gesegnet werden und in Pfarrhäusern gleichgeschlechtliche Pastoren beiderlei Geschlechts zusammenleben. Nur wenn die Kirchenleitungen der beiden Kirchen in Deutschland gemeinsam eine Position zu gesellschaftlichen Fragen beziehen und diese Position auch innerhalb der Kirchen (und damit der Mehrheit der Wähler) geteilt wird, können sie im politischen Prozess auf Akzeptanz hoffen.

Um in der demokratischen Öffentlichkeit einer pluralistischen Demokratie Gehör zu finden, gibt es für kirchliche Stellungnahmen eine Reihe von Voraussetzungen: Eine erste Voraussetzung ist, dass kirchliche Positionen dem Stand der wissenschaftlichen Diskussion entsprechen, so dass man zur Beratung in der jeweiligen Sachfrage 
die hervorragendsten Wissenschaftler im jeweiligen Fachgebiet heranzuziehen hat. Dies hat Papst Franziskus 2015 bezüglich der Umweltund Klimaprobleme in der Enzyklika Laudato Si' getan. Die Kirche muss sich damit auf der Höhe der Zeit bewegen. Eine zweite Voraussetzung ist, dass die kirchlichen Vertreter die Binnenlogik der autonomen Subsysteme der Gesellschaft, nämlich der Politik, der Wirtschaft, der Medien, der Wissenschaft usw. verstehen. Dies heißt etwa, dass man sich bei kirchlichen Stellungnahmen z. B. von (ehemaligen) Abgeordneten, Ministern, Managern, Medienvertretern etc. beraten lässt, an welcher Stelle des politischen Entscheidungsprozesses und in welcher Art eine Einflussnahme erfolgsversprechend ist. Eine dritte Voraussetzung ist, dass die Kirche nicht von oben herab, in einer Art und Weise, die keinen Widerspruch duldet oder erwartet, ihre Positionen formuliert, sondern in einen Dialog eintritt, zum Gespräch einlädt und ihre Positionen rational ohne Rekurs auf Autoritäten wie die Bibel, den Katechismus oder päpstliche Enzykliken zu begründen versucht. Denn angesichts der Tatsache, dass die Kirchen ihre Auffassungen zu Fragen der politischen Ordnung (z. B. früher Monarchie, heute Demokratie), zur Religionsfreiheit usw. gewandelt haben, werden kirchliche Positionen kritisch diskutiert. In Deutschland haben katholische Laien und Politiker vielfach eine gewisse Autonomie zu den bischöflichen Positionen eingenommen. Diese von Laien vertretenen Positionen wurden später von Bischöfen übernommen. So würde z. B. in der Gegenwart kein Bischof fordern, dass muslimische Schüler in Deutschland ausschließlich in muslimischen Schulen unterrichtet werden, wie dies früher für katholische Schüler gefordert wurde. Eine vierte Voraussetzung ist, dass die Kirche ihre Werte und Überzeugungen in die Debatte einbringt, ohne dass sie ganz konkrete und detaillierte Positionen vorzugeben versucht. Konkrete Urteile beinhalten immer empirische Zusammenhänge, die Erkenntnisgegenstand anderer Wissenschaften als der der Theologie sind. Eine fünfte Voraussetzung ist, dass die Kirche ihre ethischen Stellungnahmen nicht auf wenige Themen reduziert, sondern wie Papst Franziskus in Laudato Si' anspricht, sich auf eine umfassende Human-, Kultur- und Umweltökologie ausrichtet. Sechste Voraussetzung ist, dass in allen gesellschaftlichen Fragen, in denen die Kirche selbst unmit- 
telbar exemplarisch Handeln kann, das eigene Reden durch konkretes Handeln untermauert wird. Wenn z. B. die deutschen Bischöfe gemäß Math. 25, 35: „Ich war fremd und obdachlos und ihr habt mich aufgenommen", Solidarität mit Flüchtlingen politisch fordern, dann muss man ebenso leerstehende kirchliche Gebäude / Räume (Klöster, Kirchen, Pfarrheime, Priesterseminare) für Flüchtlinge zur Verfügung stellen - auch für jene islamischen Glaubens.

Auch Länder mit einer mehrheitlich katholischen Bevölkerung sind moderne Gesellschaften, in denen Menschen die Lehre der Kirche kritisch nach ihrem Gewissen überprüfen. Zwar nennen sich $88 \%$ der Italiener katholisch, lehnen aber zu 70\% die Sexualethik der Kirche ab. So stimmten 1974 in Italien 59\% für die zivile Ehescheidung oder 1981 68\% für ein liberales Abtreibungsrecht. ${ }^{11}$ Daher haben katholische Bischofskonferenzen in solchen Ländern nicht anders argumentativ und dialogisch um die Mehrheit der Bevölkerung zu werben, als wenn in diesen Ländern Nichtkatholiken in der Bevölkerung dominieren würden. Dies hat sich 2015 auch in der irischen Volksabstimmung über die Ehe für gleichgeschlechtliche Personen gezeigt. Auch in Spanien hat die katholische Bischofskonferenz bisher nicht verstanden, wie sie sich in der Öffentlichkeit einer pluralistischen Demokratie zu verhalten hat, und ging vielfach in Konfrontation mit dem Staat. ${ }^{12}$ Deshalb muss die Kirche nicht zuerst eine belehrende, sondern eine hörende Kirche sein. Nach Gaudium et spes Nr. 36 gibt es in modernen Gesellschaften autonome Kultursachbereiche. Die Gesetzgebung ist Aufgabe der Politiker, wobei Gaudium et spes darauf hinweist, dass es einen legitimen Pluralismus von Katholiken in der Politik (GS Nr. 43) gibt. Da bei konkreten Entscheidungen aber immer auch empirische Sachverhalte eingehen und gerade in der Politik nach einer Güterabwägung das geringere Übel gewählt werden muss, ist es ihre Aufgabe, die konkreten Entscheidungen zu fäl-

11 Vgl. „Jahrbuch für Christliche Sozialwissenschaften“ 44. Bd. (2003) zum Thema: Religion im öffentlichen Raum: Perspektiven in Europa, zu Italien: Hermann Punsmann, Ambivalenz mit Optimismus. Kontinuitäten und Wandlungen in den Äußerungen italienischer Religiösität, S. 99-114.

12 Vgl. C. C. Seidel, Spaniens neue reconquista? Re-Evangelisierungsoffensive und Religiösität in einer säkularisierten Gesellschaft?, „Jahrbuch für Christliche Sozialwissenschaften“ 44. Bd. (2003), S. 85-98. 
len. Kirchliche Amtsträger haben sich in konkreten parteipolitischen Optionen zurückzuhalten, weil es keine Zielsetzung sein kann, eine Gemeinde durch Parteipolitik zu spalten, sondern kirchliche Amtsträger haben einen Dienst an der Einheit zu vollziehen.

\section{Probleme der zunehmenden religiösen und weltanschaulichen Pluralisierung}

Die Entwicklung der religiösen Landschaft in Deutschland führt zu verschiedenen politischen Herausforderungen. Religion wird wieder zu einem Gegenstand der Politik, so dass von einem neuen Politikfeld die Rede ist: der Religionspolitik ${ }^{13}$. Welche Problembereiche sind dabei zu bearbeiten?

\subsection{Konsequenzen der schwindenden Bedeutung der etablierten Großkirchen}

Da die Evangelische und die Katholische Kirche nicht mehr 90\% der Bevölkerung organisieren, stellt sich die Frage, ob ihre gesellschaftliche Stellung nicht bedeutender geblieben ist, als es ihrer faktischen Verankerung in der Gesellschaft entspricht. Dies soll anhand von drei Problembereichen näher verdeutlich werden. Im Rahmen von Staatsleistungen (Zahlungen aus den Staatshaushalten) erhalten die beiden großen Kirchen in Deutschland zusätzlich zur Kirchensteuer zusammen fast 500 Millionen Euro im Jahr. Warum sollen konfessionslose, muslimische oder andersgläubige Steuerzahler die Gehälter katholischer und evangelischer Bischöfe und Kirchenleitungen (z. B. Domkapitel) finanzieren? Die Ablösung dieser Staatsleistungen ist seit 1919 Forderung des deutschen Verfassungsrechts, wurde aber bisher nicht realisiert. Selbst die DDR zahlte die Staatsleistungen auf niedrigem Niveau weiter. Zweitens stellt sich die Frage, ob die Kirchen nicht in gesellschaftlichen Gremien und Institutionen (z. B. den Rundfunkräten) stärker vertreten sind, als es

13 Vgl. das Themenheft Religionspolitik der „Zeitschrift für Politik“ Heft 2, 61. Jg. (2014). 
ihrer schrumpfenden Verankerung in der Bevölkerung entspricht. Hier stellt sich die Frage, ob nicht auch Vertreter muslimischer Organisationen hinzukommen sollten. Die dritte Frage betrifft die Problematik, ob nicht zumindest in einigen Regionen Deutschlands überlieferte, fast monopolartige Strukturen kirchlicher Wohlfahrtsverbände dazu führen, dass dort inzwischen ansässige konfessionslose und muslimische Bewohner praktisch nur katholische Kindergärten, Krankenhäuser und Altenheime nutzen können, weil Angebote anderer Träger regional nicht verfügbar sind. So sind z. B. im Emsland <Westniedersachsen $>$ 89\% der Kindergartenplätze in der Hand der katholischen Pfarrgemeinden.

\subsection{Integration von Minderheiten}

in die etablierten Staats-Kirchen-Beziehungen

Angesichts einer wachsenden weltanschaulichen und religiösen Pluralisierung der Gesellschaft gibt es hinsichtlich etablierter Beziehungen zwischen Staat und Kirche zwei Möglichkeiten: Die erste Möglichkeit besteht darin, die bisher auf zwei Großkirchen zugeschnittenen Staats-Kirchen-Beziehungen und die vielfältigen Kooperationen zwischen Kirche und Staat abzubauen. Diese Kooperationsbeziehungen betreffen den sozialen Sektor, in dem Caritas und Diakonie mit zusammen mehr als 1 Mill. Beschäftigten (allein Caritas 590000 in Deutschland, Volkswagen als größtes deutsches Unternehmen hat genauso viele Mitarbeiter, aber weltweit) die größten Anbieter sozialer Dienstleistungen sind. Sie betreffen zweitens den Bildungsbereich, wo in der Schule Religionsunterricht öffentliches Lehrfach ist, kirchliche Privatschulen und private kirchliche Hochschulen (z. B. für soziale Arbeit und Pflege), die staatlich unterstützt werden, die Universitäten, in denen Theologie ein Bestandteil der Wissenschaften darstellt, und die Jugend- und Erwachsenbildung, die in Akademien und Bildungshäusern stattfindet. Dies betrifft weiter kirchliche Stipendienwerke, die akademischen Nachwuchs fördern. Dies betrifft die Seelsorge in Militär, Gefängnissen, öffentlichen Krankenhäusern etc. Weiterhin betrifft dies die Entwicklungszusammenarbeit, wo Projekte kirchlicher Hilfswerke oder aber auch der internationalen Nothilfe der Caritas staatlich unterstützt werden. 
Wenn man der Auffassung ist, dass sich diese Aktivitäten bewährt haben und gesellschaftlich vorteilhaft sind, stellt sich nicht die Frage, diese etablierten Strukturen einzuschränken oder gar aufzuheben, sondern ob diese auch auf andere Gruppen, vor allem die große Gruppe der Muslime, in Deutschland ebenfalls angewandt werden könnten?

In den letzten 10-15 Jahren ist im Rahmen der deutschen Religionspolitik die Entscheidung gefallen, diese etablierten Kooperationsbeziehungen zwischen den christlichen Kirchen und dem Staat auch auf den muslimischen Bereich zu übertragen. Die institutionelle Stärkung der islamischen Bevölkerung in Deutschland wurde von den christlichen Kirchen ausdrücklich unterstützt, weil damit eine gesellschaftliche Rolle von Religion ausdrücklich anerkannt wird und ein anderes Verhältnis zur öffentlichen Rolle von Religion als etwa in Frankreich etabliert wird, wo eine striktere Trennung von Kirche und Politik herrscht. Die in diesem Sinne damit von deutschen Innenministern eingeführten Gespräche in der „Islamkonferenz" stehen aber vor der Schwierigkeit, dass die islamische Bevölkerung in Deutschland in verschiedene konfessionelle Richtungen der Sunniten, Schiiten und Alawiten gespalten ist.

In diesen Grundrichtungen gibt es wiederum verschiedene Ausprägungen, die entweder auf den jeweiligen nationalen Herkunftsländern oder unterschiedlichen religiösen Traditionen beruhen. Während es für den Staat ebenso bei der Katholischen Kirche mit den Bischöfen oder den diplomatischen Beziehungen mit dem Vatikan, wie mit den Evangelischen Bischöfen eindeutige Ansprechpartner gibt, sind Muslime auf Gemeindeebene organisiert. Die zahlenmäßig stärkste Gruppierung, die türkischen Gemeinden, werden häufig von der türkischen Religionsbehörde beeinflusst. Aus der Türkei kommen zudem Imame, die wenig von den gesellschaftlichen Verhältnissen Deutschlands wissen. Die religiöse Unterweisung erfolgt vielfach an Koranschulen, die an Moscheen angeschlossen sind, mit zu geringem Bezug zur deutschen Gesellschaft.

Daher wird seit einigen Jahren islamische Theologie an einigen deutschen Universitäten eingeführt. Um in ausreichender Zahl wissenschaftlichen Nachwuchs für die Islamische Theologie zu gewinnen, hat die mit der CDU verbundene und in der katholischen Soziallehre verankerte Konrad-Adenauer-Stiftung ein Promotionsprogramm für Doktoranden in islami- 
scher Theologie gefördert. Später wurde ein islamisches Stipendienwerk mit staatlicher Unterstützung eingerichtet. Schrittweise wird auch islamischer Religionsunterricht eingeführt. Dabei besteht ein Problem darin, wer für die islamische Seite den islamischen Theologen an den Universitäten die Lehrerlaubnis erteilt, wer dies für Religionslehrer tut und wer Lehrpläne und Religionsbücher billigt. Dies geschieht bisher in einer Grauzone..$^{14}$ Der Integrationsprozess in diesem Bereich läuft innerislamisch zum Teil sehr konfliktiv ab, weil manche islamische Theologen den Gemeindevertretern als zu liberal erscheinen.

Die Schaffung eines ,demokratieaffinen“ Islams steht vor zwei weiteren Herausforderungen: Ein erstes Problem ist die Finanzierung muslimischer Gemeinden. Bisher gibt es teilweise eine Auslandsfinanzierung, die als problematisch angesehen wird, wenn diese etwa aus Saudi-Arabien kommt und mit dem Wahhabitismus einen besonders konservativ-radikalen Islam fördert. Sinnvoll wäre es, wenn ein deutscher Islam sich auch aus Deutschland finanziert, so dass bereits eine Islamsteuer in der Öffentlichkeit diskutiert wird.

Ein zweites Problem ist die Frage der sozialen Dienstleistungen. Sollen sich die etablierten Wohlfahrtsverbände öffnen, indem etwa in katholischen Krankenhäusern und Altenheimen das Essen nach Speisevorschriften der Muslime (kein Schweinefleisch) angeboten wird, muslimische Gebetsräume eingerichtet werden und auch muslimische Mitarbeiter (z. B. wegen Sprachbarrieren) eingestellt werden? Sollen katholische Krankenhäuser auch Kopftuchtragende muslimische Krankenschwestern beschäftigen, wie dies bereits in einige Fällen geschieht? Oder soll sich ein eigener muslimischer Wohlfahrtsverband gründen, so dass etwa islamische Altenheime gegründet werden können, wo dann beispielsweise nur muslimische Frauen von Pflegerinnen und muslimische Männern von Pflegern versorgt werden? Die Lösung dieser Frage ist noch offen, so dass die große Anzahl muslimischer Flüchtlinge von christlichen wie säkularen Wohlfahrtsverbänden (z. B. Rotes Kreuz) betreut werden.

14 Vgl. M. Azzaoui, Aufdem Weg zur Anerkennung muslimischer Religionsgemeinschaften. Der religionspolitische Kompromiss in NRW zum Islamischen Religionsunterricht, „Amosinternational “ 6. Jg., Heft 1 (2012), S. 18-24. 


\subsection{Religionspolitische Konfliktfelder ${ }^{15}$}

\subsubsection{Moscheebau}

Religiöse Gemeinschaften benötigen für ihre Gottesdienste und Versammlungen Gebäude. Die Katholische Kirche hat entschieden, dass Kirchen, die nicht mehr für Gottesdienste benötigt und daher aufgegeben werden, nicht an Moscheegemeinden abgegeben werden, sondern für diese eine andere Verwendung gesucht wird. Solche Kirchen werden aber an nichtkatholische christliche Gemeinden abgegeben. In Deutschland hat es über große repräsentative Moscheebauten Diskussionen gegeben. Eine Großmoschee in Köln wurde etwa von dem bekannten katholischen Kirchenbauarchitekten Böhm geplant. In der Schweiz hat die antimuslimische Stimmung dazu geführt, dass in einer Volksabstimmung mehrheitlich ein „Minarett-Verbot" ${ }^{\text {"16 }}$ beschlossen wurde. Dies gilt aber als Verletzung der Religionsfreiheit und steht im Widerspruch zu den europäischen Menschenrechtsverträgen, die auch die Schweiz unterzeichnet hat.

\subsubsection{Beschneidung}

Bei Muslimen und Juden ist eine Beschneidung von Jungen üblich. Als dies in Deutschland als strafbare Körperverletzung aufgrund einer einzelnen Gerichtsentscheidung einer unteren Instanz problematisiert wurde, kam es zu einer Gesetzesänderung, die weiterhin eine Beschneidung ermöglicht. ${ }^{17}$ Dieses Anliegen jüdischer und muslimischer Gemeinden wurde von den beiden christlichen Kirchen in Deutschland unterstützt. ${ }^{18}$ In den USA ist die Beschneidung in weiten Bevölkerungsteilen (jenseits

15 Vgl. H. Bielefeld, Menschenrechte in der Einwanderergesellschaft. Plädoyer für einen aufgeklärten Multikulturalismus, Bielefeld 2007.

16 Vgl. A. Liedhegener, Das Feld der »Religionspolitik« - ein explorativer Vergleich der Bundesrepublik Deutschland und der Schweiz seit 1990, „Zeitschrift für Politik“ 61. Jg. (2014), S. 182-208, bes. 192 .

17 Vgl. B. E. Klein, Beschneidung im Judentum - eine Frage der Religionsfreiheit?, [in:] Religionsfreiheit. Positionen - Konflikte - Herausforderungen, Hg. H. G. Ziebertz, Würzburg 2015, S. 133-161.

18 Vgl. Th. Großbölting, Der verlorene Himmel, Glaube in Deutschland seit 1945, a.a.O., S. 228. 
von Muslimen und Juden) verbreitet. Sie gilt als vorteilhaft, weil eine geringere Übertragungsgefahr bei HIV (AIDS) bestehen soll.

\subsubsection{Weibliche Genitalverstümmelung}

Bereits aus vorislamischer Zeit stammt die Genitalverstümmelung bei Frauen. Diese wurde von Einwanderern vor allem aus Afrika nach Europa mitgebracht. Sie wird auch an hier geborenen Mädchen vollzogen. Die Einwanderer berufen sich auf die Ausübung ihrer kulturellen Traditionen und der Religionsfreiheit. In Deutschland wie in anderen europäischen Ländern gilt aber weibliche Genitalverstümmelung als Straftat und kann auch nicht mit kulturellen bzw. religiösen Rechten legitimiert werden.

\subsubsection{Schächten}

Die Speisevorschriften von Juden und Muslimen sehen vor, dass die Tiere, die sie essen dürfen, durch einen Schnitt an der Halsschlagader getötet werden. Dies widerspricht Tierschutzgesetzen, die vorsehen, dass Tiere vor ihrer Tötung z. B. durch einen Stromschlag betäubt werden müssen. Es kommt damit zu einem Konflikt zwischen Religionsfreiheit und Tierschutz. Im Sinne des Vorrangs der Religionsfreiheit vor dem Tierschutz ist das Schächten in Deutschland durch spezielle Fleischer zugelassen worden.

\subsubsection{Burkaverbot}

In Deutschland existiert kein Burkaverbot. In anderen europäischen Ländern haben Politiker, um durch antiislamische Stimmung Wahlerfolge zu erringen, ein Burkaverbot in der Öffentlichkeit thematisiert und z. T. entsprechende gesetzliche Vorschriften erlassen. ${ }^{19}$ In Deutschland würde ein generelles Burkaverbot in der Öffentlichkeit vermutlich als verfassungswidrig gelten, weil das Grundrecht Art. 2 Grundgesetz auf freie Entfaltung der Persönlichkeit verletzt würde.

19 Vgl. dazu M. Nußbaum, Die neue religiöse Intoleranz. Ein Ausweg aus der Politikder Angst, Darmstadt 2014, S. $92 \mathrm{ff}$. 


\subsubsection{Kopftuch}

Schülerinnen und Studentinnen dürfen in Deutschland - im Gegensatz zu Frankreich - in Schulen und Universitäten ein Kopftuch tragen. Umstritten war und ist die Frage, ob in der Schule eine Lehrerin ein Kopftuch tragen darf. Einerseits ist sie als Lehrerin Vertreterin eines weltanschaulich neutralen Staates. Andererseits hat sie auch das Recht auf freie Entfaltung der Persönlichkeit. Von 2004 bis 2006 erließen zahlreiche Bundesländer ein Verbot für Lehrerinnen, einen islamischen Schleier in der Schule zu tragen. Der Staat darf aber von seinen Bediensteten nur in begründeten Fällen dieses Persönlichkeitsrecht einschränken. Gesetze, die Lehrerinnen generell verbieten, ein Kopftuch zu tragen, wurden jüngst für verfassungswidrig erklärt. Viele Studentinnen der islamischen Theologie studieren mit Kopftuch. Dass Kopftuchtragen war zwar im islamischen Religionsunterricht erlaubt, Lehrer in Deutschland haben aber zwei Fächer zu unterrichten, so dass sie im Deutsch- oder Mathematikunterricht kein Kopftuch tragen durften. Nach einer Entscheidung des deutschen Verfassungsgerichts aus dem Jahr 2015 muss der Staat bei Lehrerinnen das Kopftuchtragen dulden, soweit der Schulfriede nicht massiv gestört wird. Dass Länder wie Frankreich und Belgien ein Kopftuchverbot auch für Schülerinnen erlassen haben, wertet Martha Nußbaum ${ }^{20}$ zu Recht als Ausdruck einer zunehmenden Intoleranz.

\subsubsection{Einbürgerungstest}

In den letzten Jahren ist der Zugang zur deutschen Staatsbürgerschaft erleichtert worden. Bewerber um die deutsche Staatsbürgerschaft müssen einen Einbürgerungstest absolvieren, in dem Grundwissen über deutsche Geschichte, das Grundgesetz und das politische System abgefragt wird. Über den Inhalt der Fragen gab es politische Kontroversen. Es trat die Frage auf, ob z. B. nach der Einstellung zur Homosexualität gefragt werden darf: Wie würden sie es beurteilen, wenn ihr Sohn oder ihre Tochter S. $13 f$.

M. Nußbaum, Die neue religiöse Intoleranz. Ein Ausweg aus der Politik der Angst, a.a.O., 
eine eingetragene Lebenspartnerschaft eingehen würde? Diese Fragen zur Einstellung zur Homosexualität wurden als unzulässig bewertet und nicht aufgenommen. ${ }^{21}$

\subsubsection{Familiäre soziale Kontrolle und Zwangsheiraten}

In vielen traditionellen religiösen Familien, sei es bei einigen muslimischen, sowie auch bei christlichen und vor allem evangelisch-freikirchlichen Gruppen, auch mit russlanddeutschem Hintergrund, gibt es eine strenge Erziehung und Kontrolle der Kinder. Dies betrifft überwiegend die Mädchen. Diese sollen möglichst ,jungfräulich“ in die Ehe gehen. Eltern versuchen Ihre Töchter daher nicht am gemischten Sportbzw. Schwimmunterricht oder an Klassenfahrten teilnehmen zu lassen. Sie sollen sich auch nicht außerhalb der elterlichen Kontrolle bewegen, so dass sie z. B. kein Studium außerhalb des Wohnortes der Eltern aufnehmen können. Sie sollen früh heiraten, was zur Konsequenz hat, dass teilweise ein Studium oder eine Ausbildung gar nicht aufgenommen oder abgebrochen wird. Außerdem gibt es arrangierte Ehen, bis hin zu Zwangsheiraten..$^{22}$ Der Staat hat die Schulpflicht durchzusetzen ${ }^{23}$, die Schüler sind über die Menschenrechte und die demokratische Gesellschaft $\mathrm{zu}$ informieren und es ist deutlich zu machen, dass ein Grundrecht auf Religionsfreiheit sowie auf freie Eheschließung besteht und durch den Rechtstaat garantiert wird.

\section{Medien und religiöse Überzeugungen}

Der Übergang von einer einheitlichen christlichen Religion zu zwei oder mehr christlichen Religionen hatte in Europa in der Vergangen-

${ }^{21}$ Vgl. H. Bielefeld, Menschenrechte in der Einwanderergesellschaft. Plädoyer für einen aufgeklärten Multikulturalismus, Bielefeld 2007, S. $183 \mathrm{ff}$.

${ }^{22}$ Vgl. dazu H. Bielefeld, Menschenrechte in der Einwanderergesellschaft. Plädoyer für einen aufgeklärten Multikulturalismus, a.a.O., S. $155 \mathrm{ff}$.

${ }_{23}$ Vgl. M. Nußbaum, Die neue religiöse Intoleranz. Ein Ausweg aus der Politikder Angst, a.a.O., S. $110 f$. 
heit zu erheblichen Konflikten geführt, die z. T. in längeren Kriegen (Konfessionskriege 1618-1648 in Deutschland) ausgetragen wurden. In einer wachsenden religiösen und weltanschaulichen Pluralisierung liegt damit Konfliktstoff. Auch wenn jede Religionsgemeinschaft an ihrem Wahrheitsanspruch festhält, ist es trotzdem möglich, dass verschiedene Gruppierungen sich respektieren und gegenseitig fair behandeln. Dazu können innerhalb christlicher Kirchen und Gemeinschaften ökumenische Gespräche sowie interreligiöse Dialoge beitragen.

In einer Gesellschaft der freien Meinungsäußerung ist Kritik an Religion und Kirchen, sei es an den Inhalten, an der äußeren Erscheinung wie an religiösen Führungspersonen, gestattet. Eine solche Kritik darf aber nicht den gesellschaftlichen Frieden gefährden. Deshalb ist die Menschenwürde von Personen mit anderer religiöser und weltanschaulicher Ausrichtung zu respektieren. Dies beinhaltet dann, dass die Form der Kritik und Distanzierung auch Grenzen aufweisen muss. Dies gilt nicht nur für Einzelpersonen, sondern auch für Medien. Da es in einem demokratischen Staat keine Zensur gibt, ist eine staatliche Überwachung von Medien problematisch. In Deutschland hat der deutsche Presserat im Sinne einer ethischen Selbstverpflichtung einen Pressekodex verabschiedet, worin es zu dieser Problematik heisst: „Die Presse verzichtet darauf, religiöse, weltanschauliche oder sittliche Überzeugungen zu schmähen. ${ }^{\text {“ }}{ }^{24}$ Bei Verstößen kann der Deutsche Presserat angerufen werden, der Medien auch öffentlich rügt.

\section{Schlussfolgerung}

Die zunehmende religiöse und weltanschauliche Pluralisierung führt dazu, dass Religion wieder ein wichtiger Gegenstand der Politik wird. Für viele Politikbereiche (Schulen, Universitäten, Sozialpolitik, Entwicklungspolitik, Verteidigungspolitik, Medienpolitik) fließen solche Dimensionen ein. Dabei geht es zum einen darum, dass Religionsgemeinschaften inner-

24 Siehehierzu:http://www.presserat.de/fileadmin/user_upload/Downloads_Dateien/ Pressekodex_bo_web_2015.pdf (6.11.2015). 
halb einer auf den Menschenrechten gegründeten Demokratie diese positiv anerkennen und respektieren. Zweitens geht es darum, dass Religionsgemeinschaften innerhalb des demokratischen Rechtsstaates eine konstruktive Rolle der Gesellschaftsgestaltung bilden und ihren legitimen Ort in der öffentlichen Meinungsbildung haben. Dabei ist deutlich zu sehen, dass die etablierten Staat-Kirche-Beziehungen die Konstellationen in den einzelnen Ländern prägen. Auf sie wirken weiterhin die religiöse und weltanschauliche Zusammensetzung der Bevölkerung ein. Dabei ist immer die Religionsfreiheit zu beachten, zum einen die individuelle Religionsfreiheit des Einzelnen, der sich zu einer Religionsgemeinschaft bekennt, aber sich auch vor ihr abwenden und einer anderen Kirche oder Religionsgemeinschaft zuwenden darf. Zum anderen aber auch die kollektive Religionsfreiheit, die Gruppen von Gläubigen ermöglichen muss, sich zu organisieren, Gemeinden zu bilden und die dazugehörigen Einrichtungen (wie Gotteshäuser: Kirchen, Synagogen, Moscheen, Tempel etc.) zu unterhalten. Zur Religionsfreiheit gehört aber auch, keiner Religionsgemeinschaft angehören zu müssen. Im öffentlichen Leben (z. B. bei der Besetzung von Arbeitsstellen) darf die Religionszugehörigkeit weder positiv noch negativ eine Rolle spielen. Ausnahmen sind die Religionsgemeinschaften selbst, die Angehörige ihrer eigenen Konfession beschäftigen wollen. 


\section{Summary}

\section{Religionspolitik in Deutschland nach dem Ende des Sozialismus}

Der Beitrag zeigt zunächst die gesellschaftliche Entwicklung der christlichen Konfessionen, Religionslosen sowie anderer Weltreligionen - vornehmlich Muslimen vor dem Hintergrund der Immigration und Staats-Kirchen-Beziehungen in Deutschland auf. Die religiöse Pluralität der Gesellschaft stellt eine Herausforderung für das öffentliche Wirken der katholischen, ebenso wie der evangelischen - Kirche in Deutschland dar, besonders wenn christlichen Vorstellungen in Politik und Gesellschaft Gehör verschafft werden soll. Abschließend werden beispielhaft die Bemühungen aufgezeigt, die muslimische Minderheit in die etablierten Beziehungen von Kirchen und Staat zu integrieren und dargelegt, welche Probleme dabei gelöst werden müssen.

Schlüsselwörter: Religionsfreiheit, Öffentlichkeitsauftrag der Kirchen

\section{Religious Reality in Germany after the End of the Socialism}

The article initially shows the development of the Christian confessions, non-religious and other world religions, especially Muslims, in the context of State-Church-Relations in Germany. The plurality of the society is a challenge for the public act of the Catholic and Protestant Church in Germany, especially when Christian ideas should be perceived in politics and society. Finally, the efforts to integrate the Muslim minority in the established State-Church-Relations and the challenges in this context are named.

Keywords: Religous freedom, public task oft he Church

\section{Bibliographie}

Azzaoui M., Auf dem Weg zur Anerkennung muslimischer Religionsgemeinschaften. Der religionspolitische Kompromiss in NRW zum Islamischen Religionsunterricht, „Amosinternational" 6. Jg., Heft 1 (2012), S. 18-24.

Bielefeld H., Menschenrechte in der Einwanderergesellschaft. Plädoyer für einen aufgeklärten Multikulturalismus, Bielefeld 2007.

Deutscher Presserat, Publizistische Grundsätze (Pressekodex), v. 11. März 2015, http:// www.presserat.de/fileadmin/user_upload/Downloads_Dateien/Pressekodex_bo_ web_2015.pdf (6.11.2015).

Forschungsgruppe Weltanschauungen in Deutschland, http://www:Fowid.de (10.11.2015). 
Großbölting Th., Der verlorene Himmel: Glaube in Deutschland seit 1945, GöttingenBristol, CT 2013.

Häring S., Historische Begründung und Entwicklung der Staatsleistungen an die katholische Kirche in Deutschland bis 1919, [in:] Grund und Grenzen staatlicher Religionsförderung unter besonderer Berücksichtigung des Verhältnisses von Staat und Katholischer Kirche in Deutschland, hg. von M. Pulte, A. Hense (Kirchen- und Staatskirchenrecht 17), Paderborn 2014, S. 27-44.

Klein B. E., Beschneidung im Judentum - eine Frage der Religionsfreiheit?, [in:] Religionsfreiheit. Positionen - Konflikte - Herausforderungen, Hrsg. H. G. Ziebertz, Würzburg 2015, S. 133-161.

Liedhegener A., Das Feld der "Religionspolitik“ - ein explorativer Vergleich der Bundesrepublik Deutschland und der Schweiz seit 1990, „Zeitschrift für Politik“ 61.Jg. (2014), S. 182-208.

Liedhegener A., Macht, Moral und Mehrheiten. Der politische Katholizismus in der Bundesrepublik Deutschland und den USA seit 1960, Baden-Baden 2006.

Liedhegener A., Säkularisierung als Entkirchlichung. Trends und Konjunkturen in Deutschland von der Mitte des 19. Jahrhunderts bis zur Gegenwart, [in:] Umstrittene Säkularisierung: Soziologische und historische Analysen zur Differenzierung von Religion und Politik, Hrsg. Karl Gabriel u.a., Berlin 2012, S. 481-531.

Nußbaum M., Die neue religiöse Intoleranz. Ein Ausweg aus der Politik der Angst, Darmstadt 2014.

Punsmann H., Ambivalenz mit Optimismus. Kontinuitäten und Wandlungen in den Äußerungen italienischer Religiösität, "Jahrbuch für Christliche Sozialwissenschaften" 44 (2003), S. 99-114.

Schönberger Ch., Positivität des Rechts und Naturrecht im katholischen Staatsdenken, [in:] Handbuch der Katholischen Soziallehre, Hrsg. A. Rauscher, Berlin 2008, S. 801-811.

Ratzinger J., Naturrecht, Evangelium und Ideologie in der katholischen Soziallehre, [in:] Christlicher Glaube und Ideologie, Hrsg. K. v. Bismarck, W. Dirks, Mainz 1964, S. 24-31.

Seidel C. C., Spaniens neue reconquista? Re-Evangelisierungsoffensive und Religiösität in einer säkularisierten Gesellschaft?, "Jahrbuch für Christliche Sozialwissenschaften“ 44 (2003), S. 85-98.

Themenheft Religionspolitik der „Zeitschrift für Politik”, Heft 2, 61. Jg. (2014). 